\title{
A Novel Inflammatory-Nutritional Prognostic Scoring System for Patients with Early-Stage Breast Cancer
}

\author{
Xin Hua $\mathbb{D}^{1, *}$, Fangfang Duan $\mathbb{D}^{1, *}$, Wenyu Zhai ${ }^{2}, *$, Chenge Song', Chang Jiang', Li Wang', \\ Jiajia Huang', Huanxin Lin $^{3}$, Zhongyu Yuan' \\ 'Department of Medical Oncology, State Key Laboratory of Oncology in South China, Collaborative Innovation Center for Cancer Medicine, Sun Yat- \\ sen University Cancer Center, Guangzhou, Guangdong, People's Republic of China; ${ }^{2}$ Department of Thoracic Surgery, State Key Laboratory of \\ Oncology in South China, Collaborative Innovation Center for Cancer Medicine, Sun Yat-sen University Cancer Center, Guangzhou, Guangdong, \\ People's Republic of China; ${ }^{3}$ Department of Radiotherapy, State Key Laboratory of Oncology in South China, Collaborative Innovation Center for \\ Cancer Medicine, Sun Yat-sen University Cancer Center, Guangzhou, Guangdong, People's Republic of China
}

Correspondence: Zhongyu Yuan, Department of Medical Oncology, State Key Laboratory of Oncology in South China, Collaborative Innovation Center for Cancer Medicine, Sun Yat-sen University Cancer Center, 65I Dongfeng Road East, Guangzhou, Guangdong, 5 I 0060, People's Republic of China, Email yuanzhy@sysucc.org.cn

Huanxin Lin, Department of Radiotherapy, State Key Laboratory of Oncology in South China, Collaborative Innovation Center for Cancer Medicine, Sun Yat-sen University Cancer Center, 65I Dongfeng Road East, Guangzhou, Guangdong, 510060, People’s Republic of China, Email linhx@sysucc.org.cn

*These authors contributed equally to this work

Purpose: We attempted to explore the prognostic value of baseline inflammatory and nutritional biomarkers at diagnosis in patients with early-stage breast cancer and develop a novel scoring system, the inflammatory-nutritional prognostic score (INPS).

Patients and Methods: We collected clinicopathological and baseline laboratory data of 1259 patients with early-stage breast cancer between December 2010 and November 2012 from Sun Yat-sen University Cancer Center. Eligible patients were randomly divided into training and validation cohorts ( $\mathrm{n}=883$ and 376 , respectively) in a 7:3 ratio. We selected the most valuable biomarkers to develop INPS by the least absolute shrinkage and selection operator (LASSO) Cox regression model. A prognostic nomogram incorporating INPS and other independent clinicopathological factors was developed based on the stepwise multivariate Cox regression method. Then, we used the concordance index (C-index), calibration plot, and time-dependent receiver operating characteristic (ROC) analysis to evaluate the prognostic performance and predictive accuracy of the predictive nomogram.

Results: Four inflammatory-nutritional biomarkers, including neutrophil-lymphocyte ratio (NLR), monocyte-lymphocyte ratio (MLR), prognostic nutritional index (PNI), and albumin-alkaline phosphatase ratio (AAPR), were selected using the LASSO Cox analysis to construct INPS, which remained an independent prognostic indicator per the multivariate Cox regression analysis. Patients were stratified into low- and high-INPS groups based on the cutoff INPS determined by the maximally selected rank statistics. The prognostic model for overall survival consisting of INPS and other independent clinicopathological indicators showed excellent discrimination with C-indexes of 0.825 (95\% confidence interval [CI]: 0.786-0.864) and 0.740 (95\% CI: 0.657-0.822) in the training and validation cohorts, respectively. The time-dependent ROC curves showed a higher predictive accuracy of our prognostic nomogram than that of traditional tumor-node-metastasis staging.

Conclusion: Baseline INPS is an independent indicator of OS in patients with early-stage breast cancer. The INPS-based prognostic nomogram could be used as a practical tool for individualized prognostic predictions.

Keywords: early-stage breast cancer, LASSO Cox analysis, inflammatory-nutritional biomarker, prognostic nomograms, overall survival

\section{Introduction}

Breast cancer, the most common newly diagnosed malignancy annually, is the main cause of cancer-related deaths in female patients worldwide. ${ }^{1,2}$ In recent years, advances have been made to escalate and de-escalate therapies for patients with early-stage breast cancer, such as the recommendation of adjuvant Olaparib for one year after completing local

Received: 9 September 2021 Accepted: 6 January 2022

Published: 16 January 2022 
treatment and (neo)adjuvant chemotherapy for those with high risk human epidermal growth factor receptor 2 (HER2)negative early breast cancer and germline $B R C A 1 / 2$ mutation, ${ }^{3}$ the statistically significant and clinically meaningful improvement in dual event-free survival achieved by KEYTRUDA (pembrolizumab) as (neo)adjuvant therapy in the KEYNOTE 522 trial (NCT03036488), ${ }^{4}$ the de-escalate adjuvant mono-chemotherapy with paclitaxel plus trastuzumab in HER2-positive breast cancer patients with tumors $\leq 3 \mathrm{~cm}$ and negative nodes showing excellent long-term outcomes in the APT study, ${ }^{5}$ and the exploration of trastuzumab emtansine (T-DM1) instead of trastuzumab as an adjuvant treatment for patients with HER2-positive early breast cancer and residual invasive disease after neoadjuvant chemotherapy plus HER2-targeted therapy. ${ }^{6}$ But there are still some clinical dilemmas, for example, the mutation rate of germline BRCA1/2 just accounts for $5-10 \%$ in breast cancer, most patients are still limited to chemotherapy. For non-pathological complete response (non-pCR) HER2-positive early breast cancer patients after neoadjuvant chemotherapy plus dual HER2-targeted therapy with trastuzumab and pertuzumab, the direct survival comparison between adjuvant T-DM1 and trastuzumab plus pertuzumab is poorly understood. Thus, despite above mentioned significant advances in the landscape of personalized diagnosis and therapy, breast cancer remains a huge challenge for women around the world. ${ }^{7}$ Although several genotyping prognostic models have been developed and commonly applied in clinical practice, such as Oncotype DX, MammaPrint Assay, and PAM50 gene signatures, ${ }^{8-10}$ their clinical use is limited to specific patients and not the general population. Besides, patients in developing countries are difficult to afford the high cost of the above-mentioned genomic tests due to their limited medical care insurance. Therefore, it is meaningful to identify accurate and economical prognostic biomarkers, and to develop more practical predictive models for individualized risk stratification and personalized therapeutic guidance for patients with breast cancer.

Over the years, an increasing number of studies have explored the association between nutrition, inflammation, antitumor immune response, and malignant diseases. ${ }^{11}$ Some preoperative peripheral blood parameters, which are easily obtained in routine clinical practice, could indicate the baseline immune and nutritional status of cancer patients and have been explored as prognostic biomarkers for individualized survival predictions in different types of cancers. ${ }^{12-15}$ In terms of breast cancer, several studies have shown that inflammatory-nutritional indexes, including the platelet-lymphocyte ratio (PLR), systemic inflammation score (SIS), neutrophil-lymphocyte ratio (NLR), monocyte-lymphocyte ratio (MLR), Controlling Nutritional Status (CONUT), and systemic immune-inflammation index (SII), are markedly associated with survival outcomes. ${ }^{16-21}$ Qi et al first recognized the system inflammation response index (SIRI) as a predictor for pancreatic cancer. The prognostic value of this index in patients with breast cancer was also explored in our previous study. ${ }^{22,23}$ Pretreatment serum albumin, a nutrition-related factor, and alkaline phosphatase (ALP) are markedly related to reduced inflammation and survival of cancer patients. ${ }^{24,25}$ The albumin-ALP ratio (AAPR) has been widely explored as a predictive parameter in cases of different tumors, including breast cancer. ${ }^{26}$ The nutrition-based indexes, body mass index (BMI) and prognostic nutritional index (PNI), can both reflect human nutritional status. The relationship between obesity and an elevated risk of breast tumors might promote cancer-related inflammation, and PNI could be a convenient and useful biomarker for personalized therapeutic decisions for breast cancer. ${ }^{27,28}$

Nowadays, increasing attention is being focused on the establishment of prognostic models based on abovementioned parameters for individualized survival prediction in women with breast cancer. However, no study has comprehensively studied all these inflammatory-nutritional biomarkers. We hypothesized that compared to a single biomarker, a combination of all these biomarkers might be more valuable and provide more accurate information for survival prediction. Thus, we tried to develop a novel inflammatory-nutritional prognostic scoring (INPS) system based on all available inflammatory-nutritional biomarkers and explore its relationship with the survival outcome in patients with early-stage breast cancer. Subsequently, we attempted to develop a prognostic model integrating the INPS with other clinicopathological factors for individualized prediction of survival probability in patients with early-stage breast cancer.

\section{Patients and Methods}

\section{Patient Eligibility}

Female patients with early-stage breast cancer between December 2010 and November 2012 at Sun Yat-sen University Cancer Center (SYSUCC) were retrospectively screened. Key inclusion criteria were as follows: (1) patients aged $\geq 18$ 
years; (2) patients with a pathological diagnosis of primary invasive breast cancer; (3) patients without relapse or distant metastasis (lung, liver, bone, brain) of breast cancer; (4) patients who received radical surgery; and (5) patients with available complete baseline laboratory data. The exclusion criteria were as follows: (1) pregnancy or breastfeeding; (2) presence of ductal carcinoma in situ; (3) a history of malignancy; (4) presence of severe or uncontrolled complications; (5) presence of synchronous malignancies; (6) using any medicine within 3 months before the diagnosis that could induce immune, nutritional, or inflammatory responses; and (7) presence of any chronic inflammatory disease, including autoimmune diseases. For all patients included, pathological restaging was performed according to the seventh edition of the American Joint Committee on Cancer (AJCC, 2010) criteria. Enrolled patients were randomly divided into the training and the validation cohorts in a 7:3 ratio. This study was approved by the ethics committee of SYSUCC (registration number: B2020-215-01), and the requirement of obtaining written informed consent from the patients was waived owing to the retrospective nature of current study. Patients' medical data were handled confidentially, and the study was performed following the Declaration of Helsinki.

\section{Data Collection and Classification of Variables}

Patients' clinicopathological information was manually retrieved from the electronic medical record system of SYSUCC, including age at diagnosis, menstrual status, histological type, $\mathrm{T}$ stage, $\mathrm{N}$ stage, estrogen receptor (ER) status, progesterone receptor (PR) status, human epidermal growth factor receptor 2 (HER2) status, and KI-67 index, which is assessed using routine immunohistochemical staining with the MIB1 monoclonal antibody (ZSGB-BIO, Beijing, China) according to the recommendation from the International Ki67 in Breast Cancer working group ${ }^{29}$ by at least two professional pathologists. The BMI and peripheral blood samples of patients were obtained and tested within 1 week before the diagnosis. A cutoff point of $23 \mathrm{~kg} / \mathrm{m}^{2}$ for BMI was defined as the median BMI for all patients in this study. The cut-off for ER and PR positive is defined as $\geq 1 \%$ positive cells by immune-histochemistry staining. ${ }^{30}$ HER2-positive status was recognized as a score of $3+$ or $2+$ on immunohistochemistry (IHC) with amplification of ERBB2 gene expression on fluorescence in situ hybridization (FISH) according to the American Society of Clinical Oncology/College of American Pathologists Clinical (ASCO/CAP) Practice Guidelines. ${ }^{31}$ The inflammatory-nutritional indexes were calculated using the following formulae: NLR $=\mathrm{N} / \mathrm{L}, \mathrm{PLR}=\mathrm{P} / \mathrm{L}, \mathrm{MLR}=\mathrm{M} /$ $\mathrm{L}, \mathrm{SII}=\mathrm{P} \times \mathrm{N} / \mathrm{L}, \mathrm{SIRI}=\mathrm{N} \times \mathrm{M} / \mathrm{L}, \mathrm{PNI}=\operatorname{albumin}(\mathrm{g} / \mathrm{L})+5 \times \mathrm{L}\left(10^{9} / \mathrm{L}\right)$, and AAPR $=$ albumin $(\mathrm{g} / \mathrm{L}) / \mathrm{ALP}(\mu / \mathrm{L})$ (where P: platelet count, M: monocyte count, N: neutrophil count, L: lymphocyte $\left[10^{9} / \mathrm{L}\right]$ ). Continuous laboratory parameters were classified as categorical variables according to their cutoff values determined by the maximally selected rank statistics, which were as follows: NLR (1.99), PLR (476.9), MLR (0.298), SII (739.56), SIRI (1.23), PNI (48.15), and AAPR (0.576). Regarding categorical inflammatory-nutritional indexes, CONUT was calculated based on the albumin concentration, total cholesterol concentration, and total peripheral lymphocyte count. These three variable scores were stratified into four levels (Supplementary Table 1), and the best cutoff value of 2 for CONUT was decided per our previous study. ${ }^{16,32}$ Similarly, SIS was calculated integrating serum albumin concentrations and LMR according to our previous study, ${ }^{21}$ patients with both hypoalbuminemia $(<40 \mathrm{gL}-1)$ and low LMR $(<4.44)$ were scored as 2 ; patients with either hypoalbuminemia $(<40 \mathrm{gL}-1)$ or low LMR $(<4.44)$ were scored as 1 ; patients with hyperalbuminemia ( $\geq 40 \mathrm{gL}-1)$ and $\mathrm{LMR} \geq 4.44$ were scored as 0 . A cutoff value of 1 of SIS was also determined following this study.

\section{Patients Follow-Up and Endpoints}

Follow-up information was obtained from the outpatient electronic records of SYSUCC and telephonic interviews. Patients were assessed every 3 months until 2 years, every 6 months during 2 to 5 years, and then once annually. The evaluation included routine hematological and laboratory examinations, menstrual status, breast and abdominal ultrasonography or computed tomography. X-ray and bone scans were performed annually. The primary endpoint of this study was overall survival (OS), which was defined as the time from the date of diagnosis to the date of death due to any cause or the final follow-up time. 


\section{Statistical Analysis}

Continuous variables were presented as median values with interquartile ranges (IQR), and categorical variables were listed as frequencies with percentages. Comparisons of variables between these two cohorts were performed using the Chi-square test or Fisher's exact test. We classified the candidate continuous inflammatory-nutritional indexes according to the cutoff point determined by the maximally selected rank statistics using the "maxstat" package of R software, ${ }^{33}$ with variables above and below the cutoff values scored as 1 and 0 , respectively. Considering the possibility of multicollinearity, we performed the least absolute shrinkage and selection operator (LASSO) Cox regression analysis using the "glmnet" package of R software to identify the most valuable prognostic biomarkers among all candidate inflammatorynutritional biomarkers. An L1 penalty was set in the LASSO Cox model to shrink some regression coefficients to exactly zero, and 10-fold cross-validation with minimum criteria was performed to find the optimal $\log (\lambda)$. Inflammatorynutritional biomarkers with non-zero coefficients in the LASSO analysis were incorporated to construct the novel INPS, which was calculated as follows: INPS $=$ sum (the score of every inflammatory-nutritional biomarker $\times$ corresponding regression coefficients from LASSO). On basis of the cutoff value of INPS from the maximally selected rank statistics, eligible patients in this study were stratified into the low- and high-INPS groups. Survival curves were estimated using the Kaplan-Meier method and compared using the Log rank test. The Cox proportional hazards model was used to calculate the corresponding hazard ratios (HRs) and 95\% confidence intervals (CIs). Then, we integrated the INPS with other clinicopathological variables in the univariate and multivariate Cox proportional hazards model, which were tested according to the Schoenfeld residuals, ${ }^{34}$ to identify independent predictors for OS. Variables with a $P$-value $<0.05$ in the univariate Cox model were entered into the stepwise multivariate Cox regression model, based on which a prognostic model for individualized OS predictions was established and graphically represented as a nomogram using the "rms" package of $\mathrm{R}$ software. Each subclass of these variables was correspondingly scored according to the point scale, and every patient could have a total score by adding up the score of each variable, furtherly, the OS probabilities at 1-, 3- and 5 -year could be predicted by locating total score to the survival rate scale. Subsequently, the concordance index (C-index), calibration curves, and the area under the time-dependent receiver operating characteristic (ROC) curve (AUC) were used to assess the discriminative ability and predictive performance of this prognostic model. A two-tailed $P$-value $<0.05$ was considered to indicate statistical significance. All statistical analyses were performed using R software version 4.0.1 (Vanderbilt University, Nashville, TN).

\section{Results}

\section{Patient Clinicopathological Characteristics}

A total of 1259 patients with early-stage breast cancer diagnosed at SYSUCC between December 2010 and November 2012 were enrolled in the current study. They were randomly divided into the training $(\mathrm{n}=883)$ and the validation cohorts $(n=376)$ in a 7:3 ratio. As summarized in Table 1 , the baseline clinicopathological characteristics, including the data for ten inflammatory-nutritional indexes, between the training and validation cohorts were well balanced. The median age at diagnosis of all patients was 48.0 years (IQR: 41.0-57.0 years old), and 751 (59.7\%) patients were aged less than 50 years. A total of 1060 (84.2\%) patients were pathologically diagnosed with invasive ductal carcinoma, and 586 (46.5\%) patients had a KI-67 index value $\geq 30$. Up to the last follow-up date of September 27, 2019, the median follow-up time was 78.1 months (IQR: 56.5-87.6 months), and the median OS of all enrolled patients was 78.6 months (IQR: $53.8-88.9$ months).

\section{Construction of the INPS}

A flow chart outlining INPS construction and risk stratification is provided in Figure 1. The correlation matrix for these 10 nutritional-inflammatory biomarkers (correlation coefficient $\mathrm{R}$, form -1 [blue] to 1 [red]) is presented in Figure 2A. Using the LASSO Cox regression model, we selected four inflammatory-nutritional biomarkers, namely, NLR, MLR, PNI and AAPR, of the 10 candidate parameters without zero coefficients at the optimal value -4.625423 of $\log (\lambda)$ (Figure 2B and C), and used them to construct the novel INPS for patients with early-stage breast cancer, which was calculated as follows: INPS $=0.0472271338007324 \times \mathrm{NLR}+0.369730723418473 \times \mathrm{MLR}+0.414624997751401 \times \mathrm{PNI}$ 
Table I Comparison of Baseline Clinicopathological Characteristics Between the Training and Validation Cohorts

\begin{tabular}{|c|c|c|c|c|}
\hline \multirow[t]{2}{*}{ Variables } & All & Training & Validation & \multirow[t]{2}{*}{$P$ value } \\
\hline & $N=1259$ & $\mathbf{N}=\mathbf{8 8 3}$ & $N=376$ & \\
\hline Age(years), median (IQR) & $48.0(41.0-57.0)$ & $48.0(41.0-57.0)$ & $47.5(41.0-56.0)$ & 0.395 \\
\hline Age at diagnosis & & & & 0.365 \\
\hline$\leq 50$ & $75 ।(59.7 \%)$ & $519(58.8 \%)$ & $232(61.7 \%)$ & \\
\hline$>50$ & $508(40.3 \%)$ & $364(41.2 \%)$ & 144 (38.3\%) & \\
\hline T stage ${ }^{a}$ & & & & 0.614 \\
\hline $\mathrm{TI}$ & $444(35.3 \%)$ & $320(36.2 \%)$ & $124(33.0 \%)$ & \\
\hline $\mathrm{T} 2$ & $692(55.0 \%)$ & $478(54.1 \%)$ & $214(56.9 \%)$ & \\
\hline T3 & $65(5.16 \%)$ & $43(4.87 \%)$ & $22(5.85 \%)$ & \\
\hline $\mathrm{T} 4$ & $58(4.61 \%)$ & $42(4.76 \%)$ & $16(4.26 \%)$ & \\
\hline$N$ stage $^{a}$ & & & & 0.981 \\
\hline No & $648(51.5 \%)$ & $456(51.6 \%)$ & $192(51.1 \%)$ & \\
\hline NI & $338(26.8 \%)$ & $238(27.0 \%)$ & $100(26.6 \%)$ & \\
\hline N2 & $160(12.7 \%)$ & $110(12.5 \%)$ & $50(13.3 \%)$ & \\
\hline N3 & $113(8.98 \%)$ & 79 (8.95\%) & 34 (9.04\%) & \\
\hline Menstrual status & & & & 0.316 \\
\hline Premenopausal & 745 (59.2\%) & $5 \mathrm{I} 4(58.2 \%)$ & $231(61.4 \%)$ & \\
\hline Postmenopausal & $514(40.8 \%)$ & $369(41.8 \%)$ & 145 (38.6\%) & \\
\hline Histological type & & & & 1.000 \\
\hline Others & 199 (I5.8\%) & 140 (I5.9\%) & $59(15.7 \%)$ & \\
\hline IDC & 1060 (84.2\%) & $743(84.1 \%)$ & $317(84.3 \%)$ & \\
\hline ER status & & & & 0.867 \\
\hline Negative & 354 (28.1\%) & $250(28.3 \%)$ & $104(27.7 \%)$ & \\
\hline Positive & 905 (71.9\%) & $633(71.7 \%)$ & $272(72.3 \%)$ & \\
\hline PR status & & & & 0.405 \\
\hline Negative & 452 (35.9\%) & $324(36.7 \%)$ & $128(34.0 \%)$ & \\
\hline Positive & 807 (64.1\%) & 559 (63.3\%) & $248(66.0 \%)$ & \\
\hline HER2 status & & & & 0.410 \\
\hline Negative & 886 (70.4\%) & $628(71.1 \%)$ & $258(68.6 \%)$ & \\
\hline Positive & 373 (29.6\%) & 255 (28.9\%) & 118 (31.4\%) & \\
\hline $\mathrm{Ki}-67$ index ${ }^{\mathrm{b}}$ & & & & 0.578 \\
\hline$<30$ & $673(53.5 \%)$ & 467 (52.9\%) & $206(54.8 \%)$ & \\
\hline$\geq 30$ & $586(46.5 \%)$ & $416(47.1 \%)$ & 170 (45.2\%) & \\
\hline
\end{tabular}


Table I (Continued).

\begin{tabular}{|c|c|c|c|c|}
\hline \multirow[t]{2}{*}{ Variables } & All & Training & Validation & \multirow[t]{2}{*}{$P$ value } \\
\hline & $N=1259$ & $\mathbf{N}=\mathbf{8 8 3}$ & $N=376$ & \\
\hline SIS & & & & 0.606 \\
\hline 0 & $844(67.0 \%)$ & $592(67.0 \%)$ & $252(67.0 \%)$ & \\
\hline I & $368(29.2 \%)$ & $26 \mathrm{I}(29.6 \%)$ & $107(28.5 \%)$ & \\
\hline 2 & $47(3.73 \%)$ & $30(3.40 \%)$ & $17(4.52 \%)$ & \\
\hline CONUT & & & & 0.081 \\
\hline I & 548 (43.5\%) & 402 (45.5\%) & 146 (38.8\%) & \\
\hline 2 & $436(34.6 \%)$ & $303(34.3 \%)$ & $133(35.4 \%)$ & \\
\hline 3 & 199 (15.8\%) & $125(14.2 \%)$ & 74 (19.7\%) & \\
\hline 4 & $65(5.16 \%)$ & $46(5.21 \%)$ & $19(5.05 \%)$ & \\
\hline 5 & II (0.87\%) & 7 (0.79\%) & $4(1.06 \%)$ & \\
\hline NLR, median (IQR) & $1.89(1.46-2.46)$ & I.88 (I.45-2.45) & $1.92(1.48-2.47)$ & 0.376 \\
\hline PLR, median (IQR) & $680(504-933)$ & $668(498-934)$ & $700(522-933)$ & 0.755 \\
\hline MLR, median (IQR) & $0.17(0.13-0.23)$ & $0.17(0.13-0.23)$ & $0.17(0.12-0.23)$ & 0.756 \\
\hline SII, median (IQR) & $421(307-588)$ & $416(300-586)$ & $434(325-60 I)$ & 0.270 \\
\hline SIRI, median (IQR) & $0.60(0.4 I-0.90)$ & $0.6 \mathrm{I}(0.4 \mathrm{I}-0.90)$ & $0.60(0.40-0.87)$ & 0.612 \\
\hline PNI, median (IQR) & $53.5(50.6-56.3)$ & $53.6(50.7-56.3)$ & $53.3(50.4-56.2)$ & 0.986 \\
\hline AAPR, median (IQR) & $0.71(0.57-0.86)$ & $0.7 \mathrm{I}(0.58-0.87)$ & $0.71(0.56-0.84)$ & 0.605 \\
\hline BMI, median (IQR) & $23.0(20.8-25.2)$ & 23.1 (20.9-25.3) & $22.8(20.8-25.1)$ & 0.367 \\
\hline
\end{tabular}

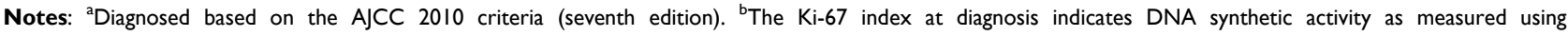
immunocytochemistry.

Abbreviations: IDC, invasive ductal carcinoma; IQR, interquartile ranges; ER, estrogen receptor; PR, progesterone receptor; HER2, human epidermal growth factor receptor 2; BMI, body mass index; NLR, neutrophil-lymphocyte ratio; PLR, platelet-lymphocyte ratio; MLR, monocyte-lymphocyte ratio; SII, systemic immune inflammation index; PNI, prognostic nutritional index; SIRI, system inflammation response index; COUNT, controlling nutritional status; SIS, system inflammation score; AAPR, album-alkaline phosphatase ratio.

$+0.402339400726211 \times$ AAPR. Then, we stratified patients in the training cohort into the low-INPS (scored $\leq 1, \mathrm{n}=698)$ and high-INPS (scored $>1, \mathrm{n}=185$ ) groups on basis of the cutoff value of INPS determined by the maximally selected rank statistics. Kaplan-Meier curves showed that patients in the high-INPS group had significantly worse survival than those in the low-INPS group (Figure 3A, $P<0.001$ ). Similarly, patients in the validation cohort were also stratified into the low-INPS (scored $\leq 1, \mathrm{n}=293$ ) and high-INPS (scored $>1, \mathrm{n}=83$ ) groups, and a significant difference in OS was also found between them (Figure 3B, $P=0.006$ ), respectively.

\section{Univariate and Multivariate Cox Analyses of OS for Breast Cancer Patients}

Results of the univariate Cox regression model in the training cohort are listed in Supplementary Table 2. Variables that achieved the predetermined significance threshold $(P<0.05)$ in the univariate Cox model, including $\mathrm{T}$ stage, $\mathrm{N}$ stage, menstrual status, histological type, ER status, PR status, HER2 status, Ki-67 index, and INPS, were entered into the stepwise multivariate Cox regression model, which revealed that the INPS remained an independent indicator of OS for patients with early-stage breast cancer ( $\mathrm{HR}=0.51 ; 95 \% \mathrm{CI}$ : 0.35-0.75, $P<0.001)$ (Figure 4). 


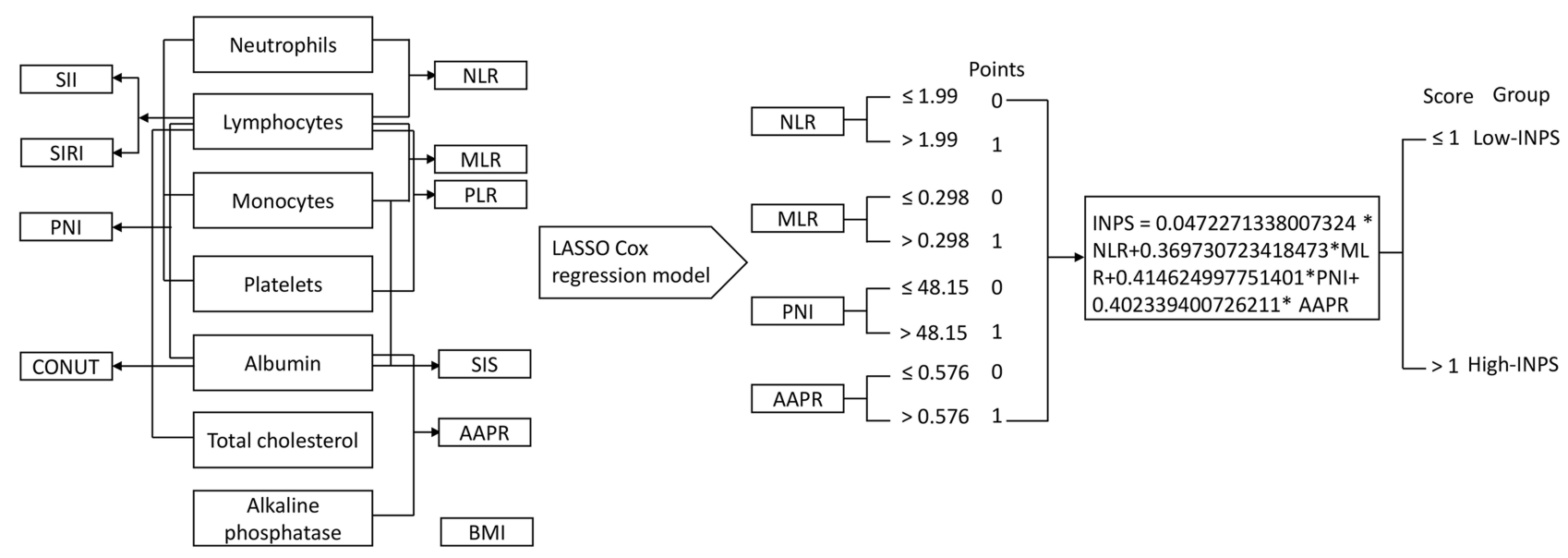

Figure I Process diagram for INPS construction and risk stratification (the asterisk *means the multiplication).

Abbreviations: INPS, inflammatory-nutritional prognostic scoring; BMI, body mass index; NLR, neutrophil-lymphocyte ratio; PLR, platelet-lymphocyte ratio; MLR, monocyte-lymphocyte ratio; SII, systemic immune inflammation index; PNI, prognostic nutritional index; SIRI, system inflammation response index; CONUT, controlling nutritional status; SIS, system inflammation score; AAPR, album-alkaline phosphatase ratio.

\section{Development of a Novel Prognostic Model Based on INPS}

Using the five independent indicators derived from the stepwise multivariate Cox analysis, namely, the $\mathrm{N}$ stage, menstrual status, histological type, Ki-67 index, and INPS, a novel prognostic nomogram for survival prediction at 1-, 3-, and 5-year was established (Figure 5A). By summing up the score of each prognostic factor subclass, a total score could be obtained for each patient at the total points line; then, the 1-, 3- and 5-year OS probabilities could be predicted by locating the total score to the survival rate scale. As we could see, patients with higher total scores demonstrated a worse individual survival probability.

\section{Assessment of Predictive Performance of the Prognostic Model}

We used the bootstrap validation method to evaluate the predictive performance of the developed prognostic model, which showed an excellent discriminative ability with a good C-index of 0.825 (95\% CI: $0.786-0.864)$ in the training cohort and 0.740 (95\% CI: 0.657-0.822) in the validation cohort. The Y-axis of the calibration curves shows the observed survival, and the X-axis shows the nomogram predicted survival. The calibration plot for the 1-, 3-, and 5-year OS represented good agreement between the nomogram predicted OS and actual OS in both the training (Figure 5B) and validation cohorts (Figure 5C). In evaluations with the time-dependent ROC curves, the prognostic accuracy of this predictive nomogram for individualized OS was much better than that of the traditional tumor-node-metastasis (TNM) stage both in the training (Figure 5D) and the validation cohorts (Figure 5E).

\section{Discussion}

In this study, we explored a novel biomarker INPS based on the baseline NLR, MLR, PNI, and AAPR at diagnosis. According to the cut-off value of INPS determined by the maximally selected rank statistics, patients were stratified into the high-INPS group and the low-INPS group. The multivariate Cox regression analysis revealed that the high-INPS was significantly related to poorer OS of early-stage breast cancer patients. Subsequently, we combined INPS with other four significant clinicopathologic variables ( $\mathrm{N}$ stage, menstrual status, histological type, Ki-67 index) identified from the multivariate Cox model to establish a prognostic nomogram, which showed excellent predictive performance and prognostic accuracy both in the training and validation cohorts.

An increasing number of studies have demonstrated that inflammation, a hallmark feature of tumors, is related to the initiation, progression, development and maintenance of malignancies. ${ }^{35,36}$ Cancer-related inflammation revolves complicated links between the tumor and inflammatory responses, and it can result in a significantly poor prognosis and failure of therapeutic response in patients with cancer. ${ }^{1,37}$ As mediators of inflammatory response, inflammatory cells play a very important part in tumor microenvironment (TME), ${ }^{36}$ and circulating inflammatory-nutritional biomarkers, 

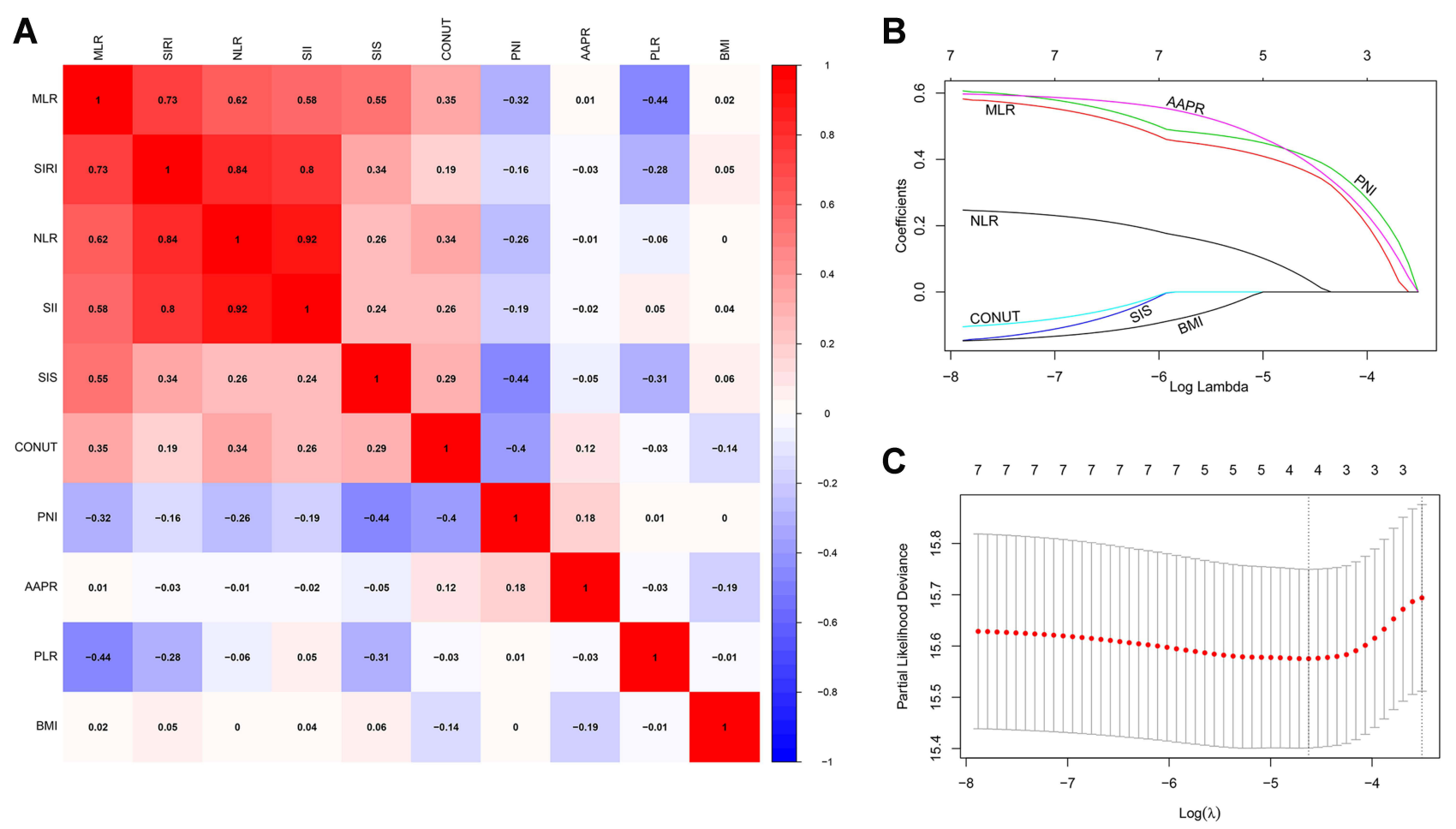

Figure 2 Construction of the INPS by using LASSO Cox regression model. (A) A correlation matrix with correlation coefficients from -I (negative correlation; blue) to I (positive correlation; red). (B) LASSO coefficient profiles of the 10 inflammatory- nutritional biomarkers. The horizontal axis (bottom) represented the log( $\lambda$ ) value of the independent variable, the horizontal axis (top) represented the number of variables with non-zero coefficient, the vertical axis represented the coefficient of the independent variable, and each curve represented the variation trajectory of the coefficient of each independent variable. (C) Ten-fold cross-validation for tuning parameter selection in the LASSO model. The dotted vertical lines were drawn at the best value of $\log (\lambda)$ by using the minimum criteria and I-SE criteria. Solid vertical lines represented partial likelihood deviance \pm SE. The intersection point of the left dotted line and the abscissa axis (bottom) showed the optimal value of log $(\lambda)$, the corresponding value in the abscissa axis (top) showed the number of variables with non-zero coefficient identified at the optimal log( $\lambda$ ).

Abbreviations: INPS, inflammatory-nutritional prognostic scoring; BMI, body mass index; NLR, neutrophil-lymphocyte ratio; PLR, platelet-lymphocyte ratio; MLR, monocyte-lymphocyte ratio; SII, systemic immune inflammation index; PNI, prognostic nutritional index; SIRI, system inflammation response index; CONUT, controlling nutritional status; SIS, systemic inflammation score; AAPR, albumin-alkaline phosphatase ratio; SE, standard error.
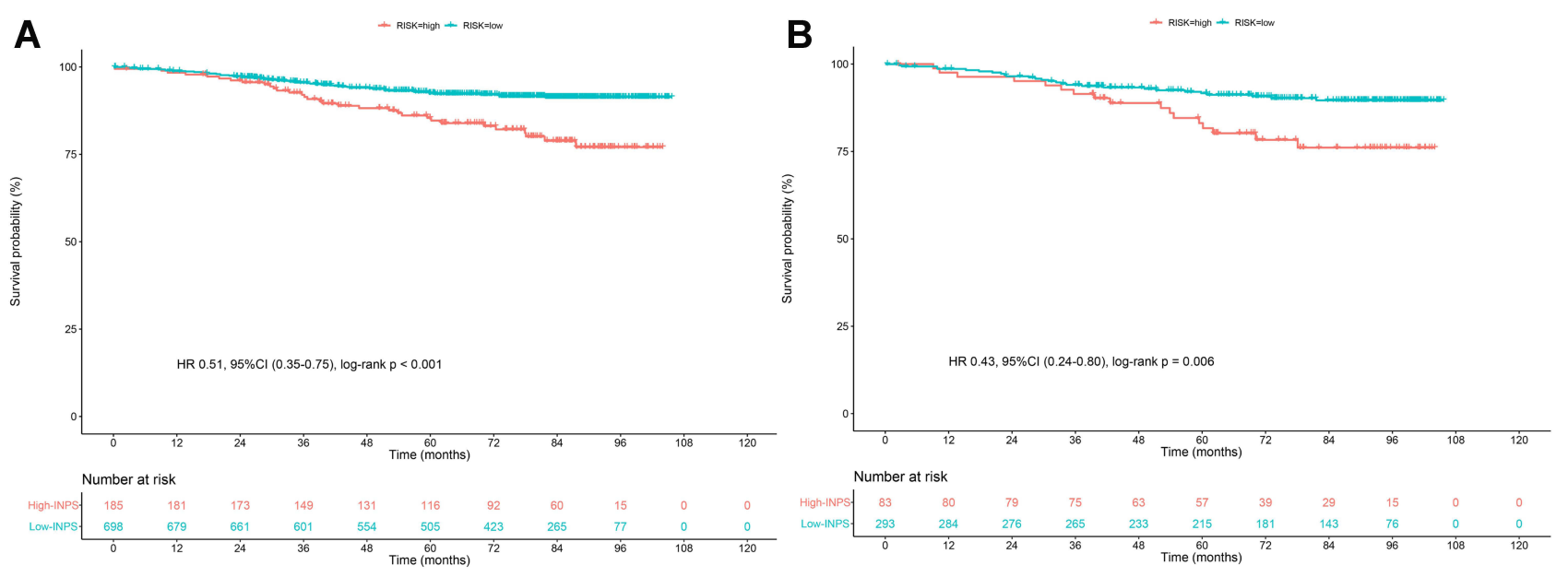

Figure 3 Survival curves obtained with Kaplan-Meier analysis between different INPS groups. The HRs reported were unadjusted. (A) Survival curves in the training cohort. (B) Survival curves in the validation cohort.

Abbreviations: INPS, inflammatory-nutritional prognostic scoring; HR, hazard ratios; Cl, confidence interval.

including PLR, NLR, MLR, CONUT, SIS, SIRI, SII, and AAPR, are significantly associated with survival and have been considered as independent predictors for breast cancer patients. ${ }^{16-18,21,23,26}$ Over the past few years, optimal predictors or prognostic models based on one or a few inflammatory-nutritional biomarkers for women with breast cancer have been 


\begin{tabular}{|c|c|c|c|c|}
\hline Variable & & Hazard ratio & $95 \%$ confidence interval $(\mathrm{Cl})$ & ) $P$ value \\
\hline \multirow[t]{4}{*}{ N stage } & No & & Reference & \\
\hline & N1 & & $2.73(1.52,4.89)$ & $<0.001^{*}$ \\
\hline & N2 & -十 & $7.34(4.19,12.84)$ & $<0.001^{*}$ \\
\hline & N3 & $\mapsto$ & $11.96(6.89,20.75)$ & $<0.001^{*}$ \\
\hline \multirow[t]{2}{*}{ Menstrual status } & pre & & Reference & \\
\hline & post & & $1.66(1.16,2.39)$ & $0.006^{*}$ \\
\hline \multirow[t]{2}{*}{ Histological type } & others & & Reference & \\
\hline & IDC & & $2.20(1.07,4.52)$ & $0.032^{*}$ \\
\hline \multirow[t]{2}{*}{ PR status } & negative & & Reference & \\
\hline & positive & & $0.73(0.51,1.05)$ & 0.088 \\
\hline \multirow[t]{2}{*}{ Ki-67 index } & $<30$ & & Reference & \\
\hline & $\geq 30$ & & $1.84(1.26,2.69)$ & $0.002^{*}$ \\
\hline \multirow[t]{2}{*}{ INPS score } & high & & Reference & \\
\hline & low & & $0.51(0.35,0.75)$ & $<0.001^{*}$ \\
\hline
\end{tabular}

Figure 4 Results of the final stepwise multivariate Cox regression analysis in the training cohort in a forest plot. The HRs reported were unadjusted, $* P<0.05$. Abbreviations: PR, progesterone receptor; IDC, invasive ductal carcinoma; INPS, inflammatory-nutritional prognostic scoring.

explored extensively, but they have limited clinical value because the weight values of individual inflammatory biomarkers to the risk score are not equal. For example, a high NLR is usually considered as an indicator of a poor prognosis, ${ }^{19,38}$ but NLR failed to show independent value in the multivariate Cox analysis in other studies when identifying patients with a pathological complete response or achievement of disease-free status. ${ }^{39,40}$ A similar dilemma has also been found in other biomarkers, such as MLR. These discrepancies could be attributed to patient heterogeneity, differences in the definitions of cutoff points, and so on.

In recent years, construction of predictive models based on multiple markers rather than a single inflammatory marker has raised great interest. Integration of multiple biomarkers could provide prognostic models with substantially higher predictive accuracy than that achieved with models based on one or a few inflammatory biomarkers. ${ }^{12,21,37}$ But most of current studies simply combined candidate inflammatory parameters with strong collinearity and correlation into a multivariate Cox regression model to identify independent predictors, which can lead to conflict between parameters and certain statistical problems. ${ }^{12}$ In this study, we performed the LASSO Cox regression analysis to include the maximal number of available inflammatory-nutritional biomarkers and effectively single out four valuable inflammatory indexes, namely, NLR, MLR, PNI, and AAPR; this approach could avoid the influence of multicollinearity to some extent.

Neutrophils play a very important role in promoting metastasis of tumor cells, and elevated neutrophil levels could create a favorable TME and inhibit activation of lymphocytes. Increasing evidence have demonstrated that cancer-related lymphocyte activation can promote immune surveillance and antitumor immune response, so the activation of lymphocyte is associated with a good prognosis in cancer patients. ${ }^{41-43}$ Monocytes, as inflammatory biomarkers, have been proven to be important regulators of the development, progression, and metastasis of tumors. ${ }^{44}$ An increased NLR value indicates a relatively elevated neutrophil level or a reduction in the lymphocyte count, which could affect the homeostasis of the TME and result in tumor progression. ${ }^{19}$ MLR, the ratio of monocytes and lymphocytes, is also significantly related to poor prognosis of breast cancer patients. ${ }^{45}$ Serum albumin, a routine item tested in clinical practice, has been explored as an independent parameter of clinical outcomes in some malignancies, including breast cancer, and a reduction in the albumin level indicates malnutrition, which might lead to a poor prognosis in cancer patients. ${ }^{24,46}$ The PNI was summed using serum albumin levels and the peripheral lymphocyte counts, which have been proven to reflect the nutritional status 
A

Points

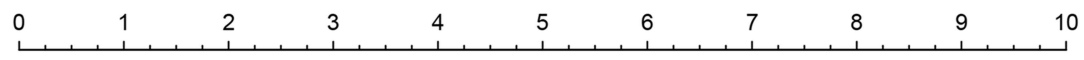

$\mathrm{N}$ stage

Menstrual status

Histological type

Ki-67 index

INPS score

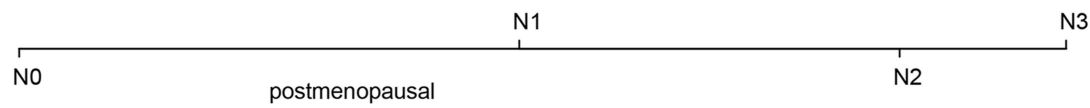

Total Points

1-year OS

3-year OS

5-year OS

premenopausal

others

$<30 \quad$ high

low

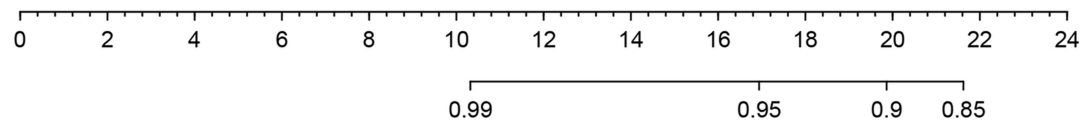

B

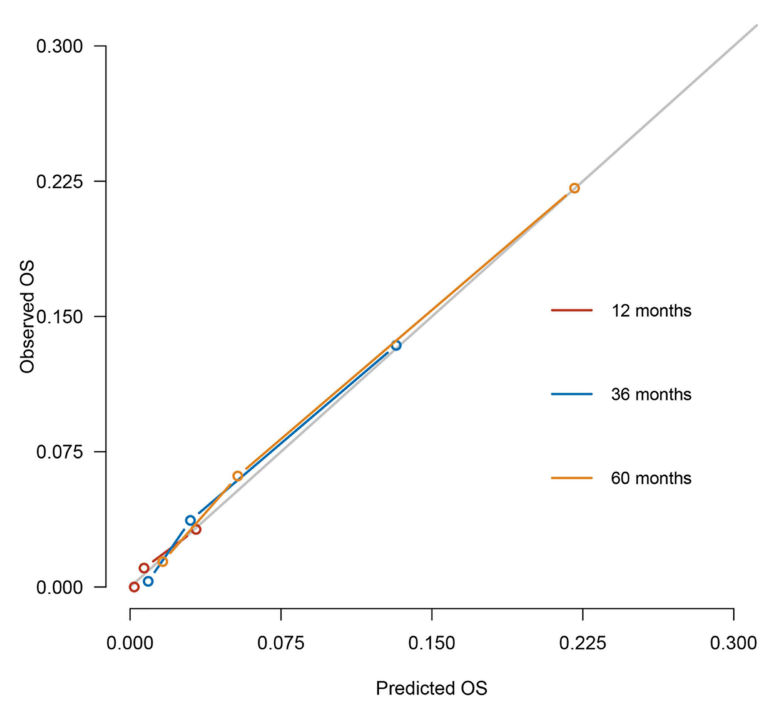

D

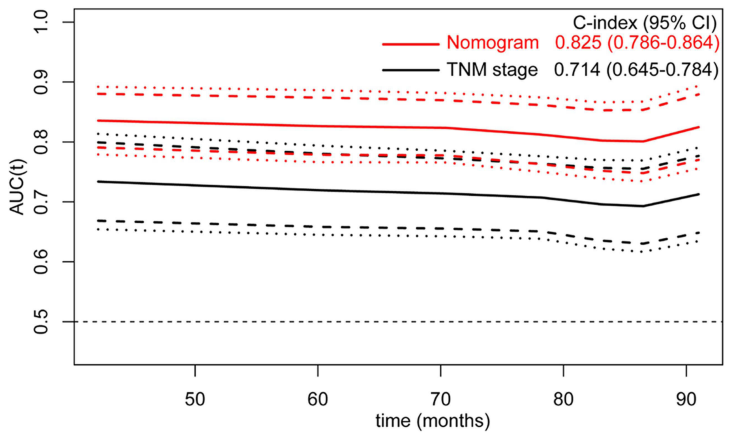

C

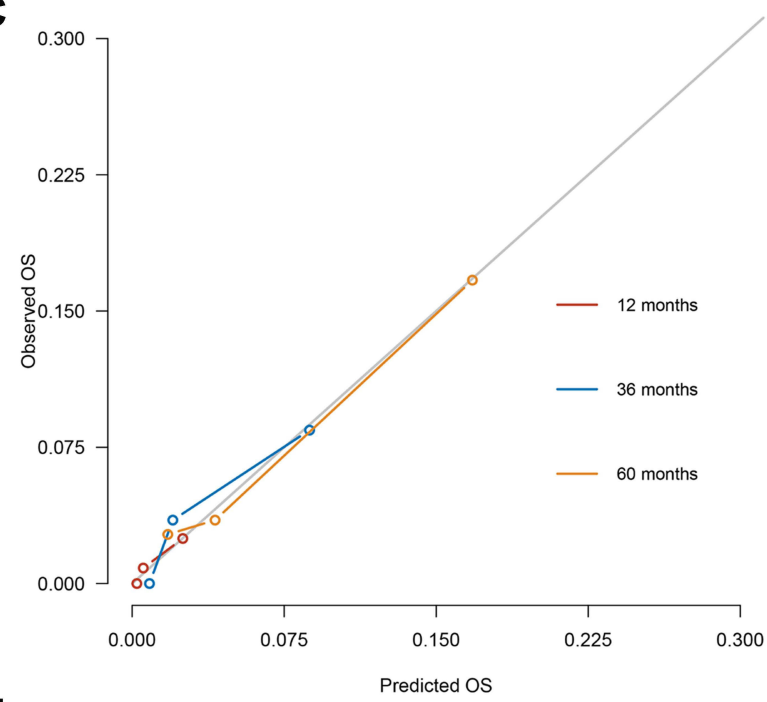

E

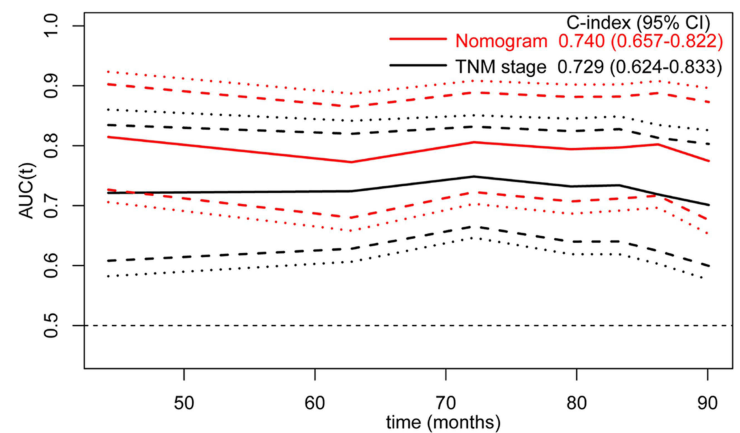

Figure 5 Development and validation of the prognostic model. (A) A nomogram of the current prognostic model for individualized survival predictions. (B) Calibration plot of the nomogram model at I-, 3-, and 5-year in the training cohort. (C) Calibration plot of the nomogram model at I-, 3-, and 5-year in the validation cohort. (D) Timeindependent ROC curves compared the predictive accuracy (C-index with its $95 \% \mathrm{Cl}$ ) of the current model (the red line) and the traditional TNM stage (the black line) in the training cohort. (E) Time-independent ROC curves compared the predictive accuracy (C-index with its $95 \% \mathrm{Cl}$ ) of the current model (the red line) and the traditional TNM stage (the black line) in the validation cohort.

Abbreviations: OS, overall survival; ROC, receiver operating characteristic; INPS, inflammatory-nutritional prognostic scoring; Cl, confidence interval. 
of patients and are associated with their survival in many studies. ${ }^{28,47}$ Elevation of the ALP level, another serum biological factor reflecting the nutritional status of humans, might represent a heavy tumor burden. ${ }^{26}$ The AAPR is calculated by dividing the serum albumin level by the serum ALP level, and its prognostic value in several cancers has been commonly recognized, ${ }^{48-50}$ similar to our previous study on patients with nonmetastatic breast cancer. ${ }^{26}$ On basis of these inflammatory-nutritional indexes, we constructed a prognostic score, which remained an independent predictor for OS in women with breast cancer after controlling other clinicopathological variables.

At present, some prognostic models are available for evaluation of breast cancer patients in clinical practice, including MammaPrint Assay, PAM50 signature and Oncotype DX. ${ }^{8-10}$ In comparison with these models, our prognostic model might be more cost-effective, accurate, and easier to be applied in primary hospitals. The prognostic value of Oncotype DX is limited to patients with hormone receptor (HR)-positive, HER2-negative breast cancer patients and the detection fee of Oncotype DX is high to nearly 20,000 CNY., ${ }^{9,51}$ Although the 70-gene MammaPrint Assay shows good stratification of chemotherapy decisions in breast cancer patients at high clinical risk, ${ }^{8}$ its $\mathrm{C}$-index reached only 0.614 in a real-word study. ${ }^{52}$ The PAM50 signature has been established on basis of the mRNA levels of 50 genes to discriminate intrinsic breast cancer subtypes, but it achieved a C-index value of only $0.63-0.67 . .^{53,54}$ Nowadays, there are an increasing number of studies on developing risk stratification or prognostic signature based on gene expression data from public databases for breast cancer, such as The Cancer Genome Atlas (TCGA), Gene Expression Omnibus (GEO) and so on, but most models are limited to preclinical explorations. ${ }^{55}$ Compared with our INPS with an average cost about 350-500 CNY, it is difficult to apply above genetic risk scores in primary hospital because the RNA sequencing requires highly professional genetic sequencing company and usually need about 20,000 CNY per examination. Considering the high economic burden and limited accuracy of above genetic models and modern assays, the TNM staging system remains to be the most frequently used tool to provide therapeutic guidance and perform follow-up assessments especially in some developing regions, in which patients have limited medical insurance. However, breast cancer patients with even the same TNM stage receiving the same treatment showed significantly different survival outcomes due to the highly heterogeneous nature of breast tumors. ${ }^{56}$ In this study, we developed a prognostic model incorporating INPS with other independent clinicopathological factors, and the C-index of this nomogram was 0.825 in the training and 0.740 in the validation cohorts, which were relatively high than that of abovementioned modern assays. Moreover, in comparison with the commonly used TNM stage, our prognostic model showed a better predictive performance according to the time-dependent ROC curves both in the training and validation cohorts. All these findings suggested that our predictive nomogram based on INPS might be a much better supplement to the traditional TNM staging, which might have a potential for individualized prognosis predictions and personalized guidance of clinical care. For example, patients with high scores on the total point scale of the graphic nomogram tended to have a high mortality, more intensive treatment, more frequent follow-up, and a more precise routine imaging monitoring, such as magnetic resonance imaging (MRI), computed tomography (CT) instead of breast/ abdominal ultrasound, might be very meaningful for patients to monitor their tumor condition and improve their outcomes as much as possible.

This study had some limitations to be noted. First, the potential selection bias due to the heterogeneity of breast cancer populations and the nature of retrospective studies is inevitable. Second, patients selected in this study were required to have completed laboratory tests of all 10 inflammatory-nutritional biomarkers, so the study population might not be representative of all females with breast cancer treated at our center. We just explored the prognostic value of baseline inflammatory-nutritional biomarkers, the dynamic change of biomarkers during subsequent anti-tumor treatment and their potential effect on survival outcomes of patients were poorly understood. Moreover, we only evaluated OS and our investigation was limited to a single center in China, so the predictive performance and extension of our prognostic nomogram are warrant further validation in multicenter or large-scale prospective cohorts. Third, we failed to explore the underlying mechanisms of INPS on breast cancer, so fundamental cytological investigations are required in future.

\section{Conclusion}

In conclusion, we constructed a convenient and economic prognostic scoring system (eg, INPS) on basis of four easily obtainable preoperative inflammatory-nutritional indexes, and the scores obtained with this system were shown to be an independent factor of OS in early-stage breast cancer. The prognostic nomogram based on the INPS showed excellent 
predictive accuracy and discriminative ability, and it may be served as a practical tool for personalized survival predictions.

\section{Data Sharing Statement}

The data analyzed in this study are available from the corresponding author (Zhongyu Yuan, E-mail: yuanzhy@sysucc. org.cn) on reasonable requests.

\section{Ethic Approval and Consent to Participate}

This study was approved by ethics committee of Sun Yat-sen University Cancer Center (registration number: B2020-21501) with a waiver of requirement of patient informed consent because we just retrospectively reviewed their medical data without impairing their health. We covered patient data confidentially and conducted this study in accordance with the Declaration of Helsinki.

\section{Acknowledgments}

We gratefully acknowledge patients and their family for all their help in enabling completion of this study. We would like to thank the native English-speaking scientists of Elixigen Company (Huntington Beach, California) for editing our manuscript.

\section{Funding}

This study was funded by the Natural Science Foundation of Guangdong Province (No. 2019A151011781), the Sci-Tech Project Foundation of Guangzhou City (No. 202002020033), and the cultivation foundation for the junior teachers in Sun Yat-sen University (No. 20ykpy164).

\section{Disclosure}

The author declare that they have no conflicts of interest for this work.

\section{References}

1. Siegel RL, Miller KD, Jemal A. Cancer statistics, 2020. CA Cancer J Clin. 2020;70(1):7-30. doi:10.3322/caac.21590

2. Sung H, Ferlay J, Siegel RL, et al. Global cancer statistics 2020: GLOBOCAN estimates of incidence and mortality worldwide for 36 cancers in 185 countries. CA Cancer J Clin. 2021;71(3):209-249. doi:10.3322/caac.21660

3. Nozawa K, Yoshimura A, Iwata H. Adjuvant olaparib in BRCA-mutated breast cancer. N Engl J Med. 2021;385(15):1439. doi:10.1056/ NEJMc2112373

4. Schmid P, Cortes J, Dent R, et al. VP7-2021: KEYNOTE-522: Phase III study of neoadjuvant pembrolizumab+ chemotherapy vs. placebo+ chemotherapy, followed by adjuvant pembrolizumab vs. placebo for early-stage TNBC. Ann Oncol. 2021;32(9):1198-1200.

5. Tolaney SM, Guo H, Pernas S, et al. Seven-year follow-up analysis of adjuvant paclitaxel and trastuzumab trial for node-negative, human epidermal growth factor Receptor 2-positive breast cancer. J Clin Oncol. 2019;37(22):1868-1875. doi:10.1200/JCO.19.00066

6. von Minckwitz G, Huang CS, Mano MS, et al. Trastuzumab emtansine for residual invasive HER2-positive breast cancer. $N$ Engl J Med. 2019;380 (7):617-628. doi:10.1056/NEJMoa1814017

7. Sachs N, de Ligt J, Kopper O, et al. A living biobank of breast cancer organoids captures disease heterogeneity. Cell. 2018;172(1-2):373-86.e10. doi:10.1016/j.cell.2017.11.010

8. Cardoso F, Van't Veer LJ, Bogaerts J, et al. 70-gene signature as an aid to treatment decisions in early-stage breast cancer. $N$ Engl J Med. 2016;375 (8):717-729. doi:10.1056/NEJMoa1602253

9. Sparano JA, Gray RJ, Makower DF, et al. Prospective validation of a 21-gene expression assay in breast cancer. $N$ Engl J Med. 2015;373 (21):2005-2014. doi:10.1056/NEJMoa1510764

10. Parker JS, Mullins M, Cheang MC, et al. Supervised risk predictor of breast cancer based on intrinsic subtypes. J Clin Oncol. 2009;27 (8):1160-1167. doi:10.1200/JCO.2008.18.1370

11. Zitvogel L, Pietrocola F, Kroemer G. Nutrition, inflammation and cancer. Nat Immunol. 2017;18(8):843-850. doi:10.1038/ni.3754

12. Wang N, Xi W, Lu S, et al. A Novel inflammatory-nutritional prognostic scoring system for Stage III gastric cancer patients with radical gastrectomy followed by adjuvant chemotherapy. Front Oncol. 2021;11:650562. doi:10.3389/fonc.2021.650562

13. Zhang N, Jiang J, Tang S, Sun G. Predictive value of neutrophil-lymphocyte ratio and platelet-lymphocyte ratio in non-small cell lung cancer patients treated with immune checkpoint inhibitors: a meta-analysis. Int Immunopharmacol. 2020;85:106677. doi:10.1016/j.intimp.2020.106677

14. Rebuzzi SE, Signori A, Banna GL, et al. Inflammatory indices and clinical factors in metastatic renal cell carcinoma patients treated with nivolumab: the development of a novel prognostic score (Meet-URO 15 study). Ther Adv Med Oncol. 2021;13:17588359211019642. doi:10.1177/ 17588359211019642 
15. Templeton AJ, McNamara MG, Šeruga B, et al. Prognostic role of neutrophil-to-lymphocyte ratio in solid tumors: a systematic review and meta-analysis. J Natl Cancer Inst. 2014;106(6):dju124. doi:10.1093/jnci/dju124

16. Huang ZZ, Song CG, Huang JJ, et al. Prognostic significance of the Controlling Nutritional Status (CONUT) score in surgically treated breast cancer patients. Gland Surg. 2020;9(5):1370-1379. doi:10.21037/gs-20-294

17. Hua X, Long ZQ, Zhang YL, et al. Prognostic value of preoperative systemic immune-inflammation index in breast cancer: a propensity score-matching study. Front Oncol. 2020;10:580. doi:10.3389/fonc.2020.00580

18. van der Willik KD, Koppelmans V, Hauptmann M, Compter A, Ikram MA, Schagen SB. Inflammation markers and cognitive performance in breast cancer survivors 20 years after completion of chemotherapy: a cohort study. Breast Cancer Res. 2018;20(1):135. doi:10.1186/s13058-018$1062-3$

19. Ethier JL, Desautels D, Templeton A, Shah PS, Amir E. Prognostic role of neutrophil-to-lymphocyte ratio in breast cancer: a systematic review and meta-analysis. Breast Cancer Res. 2017;19(1):2. doi:10.1186/s13058-016-0794-1

20. Ma Y, Zhang J, Chen X. Lymphocyte-to-monocyte ratio is associated with the poor prognosis of breast cancer patients receiving neoadjuvant chemotherapy. Cancer Manag Res. 2021;13:1571-1580. doi:10.2147/CMAR.S292048

21. Huang ZZ, Hua X, Song CG, et al. The prognostic prediction value of systemic inflammation score and the development of a nomogram for patients with surgically treated breast cancer. Front Oncol. 2020;10:563731. doi:10.3389/fonc.2020.563731

22. Qi Q, Zhuang L, Shen Y, et al. A novel systemic inflammation response index (SIRI) for predicting the survival of patients with pancreatic cancer after chemotherapy. Cancer. 2016;122(14):2158-2167. doi:10.1002/cncr.30057

23. Hua X, Long ZQ, Huang X, et al. The preoperative systemic inflammation response index (SIRI) independently predicts survival in postmenopausal women with breast cancer. Curr Probl Cancer. 2020;44(4):100560. doi:10.1016/j.currproblcancer.2020.100560

24. Gupta D, Lis CG. Pretreatment serum albumin as a predictor of cancer survival: a systematic review of the epidemiological literature. Nutr $J$. 2010;9:69. doi:10.1186/1475-2891-9-69

25. Li D, Lv H, Hao X, Hu B, Song Y. Prognostic value of serum alkaline phosphatase in the survival of prostate cancer: evidence from a meta-analysis. Cancer Manag Res. 2018;10:3125-3139. doi:10.2147/CMAR.S174237

26. Long ZQ, Hua X, Zhang WW, et al. Prognostic impact of the pretreatment albumin to alkaline phosphatase ratio for nonmetastatic breast cancer patients. Cancer Manag Res. 2019;11:4809-4814. doi:10.2147/CMAR.S200759

27. Moore SC, Playdon MC, Sampson JN, et al. A metabolomics analysis of body mass index and postmenopausal breast cancer risk. $J$ Natl Cancer Inst. 2018;110(6):588-597. doi:10.1093/jnci/djx244

28. Chen L, Bai P, Kong X, et al. Prognostic Nutritional Index (PNI) in patients with breast cancer treated with neoadjuvant chemotherapy as a useful prognostic indicator. Front Cell Develop Biol. 2021;9:656741. doi:10.3389/fcell.2021.656741

29. Dowsett M, Nielsen TO, A'Hern R, et al. Assessment of Ki67 in breast cancer: recommendations from the International Ki67 in breast cancer working group. $J$ Natl Cancer Inst. 2011;103(22):1656-1664. doi:10.1093/jnci/djr393

30. Allison KH, Hammond MEH, Dowsett M, et al. Estrogen and progesterone receptor testing in breast cancer: ASCO/CAP guideline update. J Clin Oncol. 2020;38(12):1346-1366. doi:10.1200/JCO.19.02309

31. Woo JW, Lee K, Chung YR, Jang MH, Ahn S, Park SY. The updated 2018 American Society of Clinical Oncology/College of American Pathologists guideline on human epidermal growth factor receptor 2 interpretation in breast cancer: comparison with previous guidelines and clinical significance of the proposed in situ hybridization groups. Hum Pathol. 2020;98:10-21. doi:10.1016/j.humpath.2020.01.003

32. Hirahara N, Tajima Y, Fujii Y, et al. Controlling Nutritional Status (CONUT) as a prognostic immunonutritional biomarker for gastric cancer after curative gastrectomy: a propensity score-matched analysis. Surg Endosc. 2019;33(12):4143-4152. doi:10.1007/s00464-019-06723-z

33. Hothorn T, Zeileis A. Generalized maximally selected statistics. Biometrics. 2008;64(4):1263-1269. doi:10.1111/j.1541-0420.2008.00995.x

34. Wileyto EP, Li Y, Chen J, Heitjan DF. Assessing the fit of parametric cure models. Biostatistics (Oxford, England). 2013;14(2):340-350. doi:10.1093/biostatistics/kxs043

35. Velazquez-Caldelas TE, Alcalá-Corona SA, Espinal-Enríquez J, Hernandez-Lemus E. Unveiling the link between inflammation and adaptive immunity in breast cancer. Front Immunol. 2019;10:56. doi:10.3389/fimmu.2019.00056

36. Diakos CI, Charles KA, McMillan DC, Clarke SJ. Cancer-related inflammation and treatment effectiveness. Lancet Oncol. 2014;15(11):e493-503. doi:10.1016/S1470-2045(14)70263-3

37. Li WZ, Hua X, Lv SH, et al. A scoring system based on nutritional and inflammatory parameters to predict the efficacy of first-line chemotherapy and survival outcomes for De Novo metastatic Nasopharyngeal carcinoma. J Inflamm Res. 2021;14:817-828. doi:10.2147/JIR.S296710

38. Kim JH, Son NH, Lee JS, et al. Time-sequencing of the neutrophil-to-lymphocyte ratio to predict prognosis of triple-negative breast cancer. Cancers (Basel). 2021;13:14. doi:10.3390/cancers13143472

39. Ligorio F, Fucà G, Zattarin E, et al. The pan-immune-inflammation-value predicts the survival of patients with Human Epidermal Growth Factor Receptor 2 (HER2)-positive advanced breast cancer treated with first-line taxane-trastuzumab-pertuzumab. Cancers. 2021;13:8. doi:10.3390/ cancers 13081964

40. Şahin AB, Cubukcu E, Ocak B, et al. Low pan-immune-inflammation-value predicts better chemotherapy response and survival in breast cancer patients treated with neoadjuvant chemotherapy. Sci Rep. 2021;11(1):14662. doi:10.1038/s41598-021-94184-7

41. Tsai CY, Hsieh SC, Liu CW, et al. Cross-talk among polymorphonuclear neutrophils, immune, and non-immune cells via released cytokines, granule proteins, microvesicles, and neutrophil extracellular trap formation: a novel concept of biology and pathobiology for neutrophils. Int J Mol Sci. 2021;22(6). doi:10.3390/ijms22063119

42. Denkert C, Loibl S, Noske A, et al. Tumor-associated lymphocytes as an independent predictor of response to neoadjuvant chemotherapy in breast cancer. J Clin Oncol. 2010;28(1):105-113. doi:10.1200/JCO.2009.23.7370

43. Yang L, Liu Q, Zhang X, et al. DNA of neutrophil extracellular traps promotes cancer metastasis via CCDC25. Nature. 2020;583(7814):133-138. doi:10.1038/s41586-020-2394-6

44. Olingy CE, Dinh HQ, Hedrick CC. Monocyte heterogeneity and functions in cancer. J Leukoc Biol. 2019;106(2):309-322. doi:10.1002/ JLB.4RI0818-311R

45. Wang Y, Huang G, Li Z. Prognostic significance of inflammatory biomarkers in patients with breast cancer skeletal metastases. Cancer Manag Res. 2020;12:11463-11475. doi:10.2147/CMAR.S277291 
46. Liu X, Meng QH, Ye Y, Hildebrandt MA, Gu J, Wu X. Prognostic significance of pretreatment serum levels of albumin, LDH and total bilirubin in patients with non-metastatic breast cancer. Carcinogenesis. 2015;36(2):243-248. doi:10.1093/carcin/bgu247

47. Hua X, Long ZQ, Huang X, et al. The Value of Prognostic Nutritional Index (PNI) in predicting survival and guiding radiotherapy of patients with T1-2N1 breast cancer. Front Oncol. 2019;9:1562. doi:10.3389/fonc.2019.01562

48. Tian G, Li G, Guan L, Yang Y, Li N. Pretreatment albumin-to-alkaline phosphatase ratio as a prognostic indicator in solid cancers: a meta-analysis with trial sequential analysis. Int J Surg. 2020;81:66-73. doi:10.1016/j.ijsu.2020.07.024

49. Zhou S, Wang H, Jiang W, Yu Q, Zeng A. Prognostic value of pretreatment albumin-to-alkaline phosphatase ratio in extensive-disease small-cell lung cancer: a retrospective cohort study. Cancer Manag Res. 2020;12:2015-2024. doi:10.2147/CMAR.S247967

50. Li S, Lu S, Liu X, Chen X. Association between the pretreatment albumin-to-alkaline phosphatase ratio and clinical outcomes in patients with bladder cancer treated with radical cystectomy: a retrospective cohort study. Front Oncol. 2021;11:664392. doi:10.3389/fonc.2021.664392

51. Poorvu PD, Gelber SI, Rosenberg SM, et al. Prognostic impact of the 21-gene recurrence score assay among young women with node-negative and node-positive ER-Positive/HER2-negative breast cancer. J Clin Oncol. 2020;38(7):725-733. doi:10.1200/JCO.19.01959

52. Ibraheem A, Olopade OI, Huo D. Propensity score analysis of the prognostic value of genomic assays for breast cancer in diverse populations using the National Cancer Data Base. Cancer. 2020;126(17):4013-4022. doi:10.1002/cncr.32956

53. Gnant M, Sestak I, Filipits M, et al. Identifying clinically relevant prognostic subgroups of postmenopausal women with node-positive hormone receptor-positive early-stage breast cancer treated with endocrine therapy: a combined analysis of ABCSG-8 and ATAC using the PAM50 risk of recurrence score and intrinsic subtype. Ann Oncol. 2015;26(8):1685-1691. doi:10.1093/annonc/mdv215

54. Wallden B, Storhoff J, Nielsen T, et al. Development and verification of the PAM50-based Prosigna breast cancer gene signature assay. BMC Med Genomics. 2015;8:54. doi:10.1186/s12920-015-0129-6

55. Yuan J, Duan F, Zhai W, et al. An aging-related gene signature-based model for risk stratification and prognosis prediction in breast cancer. Int $J$ Women's Health. 2021;13:1053-1064. doi:10.2147/IJWH.S334756

56. Yersal O, Barutca S. Biological subtypes of breast cancer: prognostic and therapeutic implications. World J Clin Oncol. $2014 ; 5(3): 412-424$. doi:10.5306/wjco.v5.i3.412

Journal of Inflammation Research

\section{Publish your work in this journal}

The Journal of Inflammation Research is an international, peer-reviewed open-access journal that welcomes laboratory and clinical findings on the molecular basis, cell biology and pharmacology of inflammation including original research, reviews, symposium reports, hypothesis formation and commentaries on: acute/chronic inflammation; mediators of inflammation; cellular processes; molecular mechanisms; pharmacology and novel anti-inflammatory drugs; clinical conditions involving inflammation. The manuscript management system is completely online and includes a very quick and fair peer-review system. Visit http://www.dovepress.com/testimonials.php to read real quotes from published authors. 